\title{
Calidad de vida y beneficio clínico con tratamiento radioterápico
}

\author{
C. Silva Rodríguez
}

\section{Introducción}

Los avances en la oncología han hecho posible que existan terapéuticas con gran potencial curativo, por lo que en muchos casos el cáncer puede tratarse de una enfermedad crónica.

No podemos, para valorar la efectividad de los tratamientos, medir sólo parámetros como: morbilidad, intervalo libre de enfermedad, supervivencia, etc, sino que hay que tener en cuenta los problemas funcionales y psicosociales a los que se enfrentan los pacientes en sus diversas etapas' ${ }^{1}$. Así nace el concepto de calidad de vida.

En la práctica clínica utilizamos el índice de Performance Status de Karnosfsky para algunos equivalente a calidad de vida y para otros poco fiable ${ }^{2}$, que nos ayuda a tomar decisiones terapéuticas, también los oncólogos radioterapeutas utilizamos los grados de toxicidad agudos-crónicos (RTOG versión 12-12-2003).

Se ha suscitado un interés enorme desde que la reunión de los institutos nacionales del cáncer recomendó que los recursos económicos se dieran en relación a tres variables: supervivencia, morbilidad y calidad de vida.

Sin embargo se trata de un concepto tan complejo y multidimensional que no resulta fácil ni siempre fiable su valoración, por la gran heterogeneidad de las poblaciones y el impacto que supone sobre los distintos individuos enfrentarse al diagnóstico, los tratamientos y sus secuelas.

Se han desarrollado múltiples herramientas y escalas para su valoración ${ }^{3}$, pero si podemos afirmar que no existe un instrumento único o específico de cáncer que pueda considerarse como patrón $n^{4}$.

Servicio de Oncología Radioterápica Centro Oncológico de Galicia

\section{Objetivos}

- Determinar el beneficio clínico-calidad de vida de la radioterapia en el tratamiento conservador de órganos (laringe, mama, ano, vejiga).

- Determinar la influencia de la radioterapia en la calidad de vida en pacientes en estadios avanzados.

\section{Tratamiento conservador de laringe}

Supone la conservación de funciones tan vitales como deglución, fonación, y apariencia estética.

Desde finales de los 70 principios de los 80 se intenta la preservación de la laringe introduciendo una nueva modalidad terapéutica: la quimioterapia. Los porcentajes de conservación laringea con radioquimioterapia concomitantes en Ca laringe avanzados son hasta del $88 \%$ a dos años 3 .

Se han realizado numerosos estudios de calidad de vida en estos pacientes utilizando el cuestionario EORTC-QLQC30 y su módulo de cabeza y cuello6,7, durante el primer año se observaba un detrimento en la calidad de vida, a partir del segundo año la tendencia es la mejoría hasta los niveles pretratamiento. Los aspectos que se recuperan con más dificultad son: xerostomía, sensibilidad y sexualidad. Donde había más beneficio es a nivel emocional $\left.\right|^{8,9,10}$.

\section{Tratamiento conservador de mama}

Está ampliamente demostrado que la cirugía conservadora mas radioterapia es equivalente en cuanto a supervivencia a la mastectomía radical ${ }^{11}$. La mama es un órgano con un condicionante importante en la psicología de la mujer, por ello se utilizan escalas concretas que miden el impacto de los tratamientos sobretodo a nivel de efectos psicológicos y de la sexualidad (EORTC BR23).

En los numerosos estudios realizados respecto a la calidad de vida influyen no sólo aspectos terapéuticos sino otros como la edad, estatus social ${ }^{12}$. También que la cirugía y la quimioterapia tienen un impacto negativo en la calidad de vida que la radioterapia, la astenia es el síntoma que condiciona más la funcionalidad de las pacientes durante el tratamiento.

Inicialmente el impacto psicológico del diagnóstico, la ex- 
pectativa de futuro se imponen sobre factores como la estética, la sexualidad, etc ${ }^{13}, 14$, con el tiempo la calidad de estas pacientes globalmente es buena y el tratamiento conservador aporta beneficios sobretodo en mejor imagen corporal y mayor disfrute de las relaciones sexuales ${ }^{15,16}$.

\section{Tratamiento conservador ano}

El tratamiento estándar en el cáncer anal es radioterapia y quimioterapia simultanea, este se ha impuesto sobre amputación abdominoperineal (ABP) por la conservación del esfínter. La calidad de vida no sólo va a depender de la conservación sino de la conservación del esfínter ${ }^{17}$.

Para su medición se utilizan unos módulos específicos: síntomas gastrointestinales, problemas de defecación, pérdida de peso y grado de satisfacción por la conservación del esfínter.

Los resultados de los estudios fueron la funcionalidad subóptima del esfínter en un 50\% de los pacientes pero el $71 \%$ están satisfechos por la conservación ${ }^{18}$. El principal problema es la diarrea tras la irradiación pélvica, la funcionalidad en el resto de las esferas es aceptable a excepción de la sexual que aparece claramente afectada en los varones.

\section{Tratamiento conservador vejiga}

En estadios iniciales (T2a, T2b, NOMO) el tratamiento con RTU, quimioterapia y radioterapia concomitantes, se consigue la conservación de la vejiga en un $75 \%$ de pacientes con una supervivencia global del $64 \%$ a 4 años ${ }^{19}$. Pese a la toxicidad, $6 \%$ problemas de flujo miccional, un $15 \%$ urgencia miccional, y un $19 \%$ problemas de control del esfínter. La mayoría de los pacientes refiere una función vesical aceptable, los varones mantienen una buena función sexual y globalmente la calidad de vida es buena.

\section{Radioterapia paliativa en pacientes con enfermedad avanzada}

Los objetivos son: alivio sintomático rápido y duradero y producir el menor discomfort e interferencia en el estilo de vida del paciente.

Es importante:

- Definir los síntomas atribuibles a una localización tumoral concreta que sea susceptible de ser irradiada.

- Diseñar esquemas realizables en pocos días y con dosis totales escasamente tóxicas.

- Una correcta comunicación con el paciente y su familia sobre el verdadero objetivo del tratamiento.

\section{Síntomas}

- Dolor: Se ha comprobado que se consigue una remisión del dolor en un $75 \%$ de los pacientes con metástasis óseas sintomáticas, en un $50 \%$ se consigue el alivio completo.

Las dosis que se utilizan son: $8 \mathrm{~Gy}, 5$ por $4 \mathrm{~Gy}, 10$ por 3 Gy, sin existir diferencias significativas a favor de ninguno de los fraccionamientos utilizados. Cuando son numerosas las metástasis óseas sintomáticas se puede realizar radiación hemicorporal (6 Gy hemicuerpo superior y 8 Gy hemicuerpo inferior) ${ }^{20}$

- Neurológicos: Los tumores sólidos desarrollan metástasis en SNC en un 25\%. La radioterapia holocraneal consigue mejoría de los síntomas neurológicos entre el $70-80 \%$ con supervivencia media de 4-5 meses. Con una toxicidad míni$\mathrm{ma}^{21}$.

- Respiratorios: Disnea, tos, hemoptisis, SVCS. Un 30$40 \%$ de los pacientes tratados con radioterapia paliativa presentan estos síntomas. El mayor beneficio se tiene en el síndrome de vena cava superior y hemoptisis con un $80 \%$ de mejoría.

- Síndrome de compresión medular: Un 5\% de los pacientes con cáncer avanzado presentan este síndrome. Debe tratarse de forma inmediata para procurar la recuperación funcional, y hay una escasa toxicidad neurológica.

\section{Conclusiones}

- Las escalas y mediciones para evaluar la calidad de vida tienden a la evaluación clínica, terapéutica y psicológica para estudios puntuales en determinados períodos de la enfermedad.

- En estos aspectos esta claro el beneficio clínico de los tratamiento radioterápicos tanto con intención radical como paliativa.

- Realmente carecemos de un método que mida o evalúe este aspecto en toda su complejidad.

\section{Bibliografía}

1. Aaronson NK. Methodologic issues in assessing the quality of life of cancer patiens. Cancer 1991; 67: 844-50.

2. Bloom JR. Quality of life after cancer. A poicy perspective. Cancer 1991; 67:855-8.

3. Cella DF, Tulsky SD, Gray $G$ et al. The functional assessment of cancer therapy scale; development and validation of the General measure. J Clin Oncol 1993; 11(3):570-9.

4. Wiklund I, Kalberg J. Evaluation of quality of life in clinical trials. Controlled clinical trials 1991; 12:204s-16s.

5. N Engl J Med 2003, 27349 (22).

6. Hanna E, Sherman A, Cash D, Adams D, et al. Quality of life for patiens following total laryngectomy vs chemoradiation for laryngeal preservation. Arch otolaryngol Head Neck Surg. 2004 Jul; 130 (7): 875-9.

7. Bjordal K, Kaasa S, et al. Int J Radiat Oncolo Biol Phys. 1994 Mar 1; 28(4): 847-56.

8. Harwood AR, Rwlinson E. The quality of life of patient following treatment for laryngeal cancer. Int Radiat Oncol Biol Phys 1983: Mar (9) 335-338.

9. Hammerlid E, Silander E, Hornestam L, Sullivan M. Health-related quality of life three years after dignosis of head and neck cancer- a longitudinal study. Head Neck 2001 Feb; 23(2):11325.

10. Muller R, Paneff J, Kollner V, Koch R. Quality of life of patients with laryngeal carcinoma: a post-treatment study. Arch Otorrinolayngol. 2001 Aug:258 (6): 276-80.

11. Fisher. N Engl J Med 1995 Nov 30; 333(22): 1456-61. 
12. Dow KH, Lafferty P. Quality of life, survivorship and psychosocial adjustment of young women with breast cancer after breast-conseving surgery and radiation therapy. Oncol Nurs forum 2000 Nov-Dec; 27 (10):1555-64.

13. Goodwin PJ, Black JT, Bordeleau U Ganz PA. Health-related quality of life measurement in randomized clinical trials in breast cancer-taking stock. J Nat Cancer Inst 2003 Feb 19;95 (4): 263-81.

14. Ganz PA, Kwan L, et al. Quality of life at the end of primary treatment of breast cancer: first results from the moving beyond cancer randomized trial. J Nat Cancer Inst 2004 Mar 3, 96 (5):376-87.

15. Whelan TJ, Levine $M$, et al. The effects of radiation therapy on quality of life of women with breast carcinoma; results of a randomized trial. Cancer vol 88 (10) (pp 2260-2266), 2000.

16. Carlsson $M$, Arman $M$, et al. Evaluation of quality of life/life satisfaction in woman with breast cancer in complementary an conventional care. Acta Oncol. 2004; 43 (1):27-34
17. Vordermark $D$, Sailer $M$, et al. Curative-intent radiation therapy in anal carcinoma: quality of life and sphincter function. Radiotherapy Oncology. Vol 52(3) (pp239-243). 1999.

18. Allal AS, Sprangers MA, et al. Assessment of long-term quality of life in patients with anal carcinoma treated by radiotherapy with or without chemotherapy. Br J Cancer 1999 Jul; 80(10): 1588-94.

19. Michaelson MD, Shipley WU, et al. Selective bladder preservation for muscle-invasive transitional cell carcinoma of the urinary bladder. Br J Cancer (2004) 90, 578-581.

20. Poulter CA, Cosmatos D, et al. A report of RTOG 8206: a phase III study of wheter the addition of single dose hemibody irradiation is more effective than local field irradiation alone in the treatment of symptomatic osseus metastases. Int J Radiat Oncol Biol Phys 1992; 23:207-14.

21. Kim $M$, Bernstein $M$. Current treatment of cerebral metastases. Curr Opin Neurol 1996; $9: 414-8$ 